\title{
Modeling of random anisotropic elastic media and impact on wave propagation
}

\author{
Quang-Anh Ta — Didier Clouteau — Régis Cottereau \\ * École Centrale Paris, Laboratoire MSSMat (UMR 8579) \\ Grande Voie des Vignes, F-92295 Châtenay-Malabry cedex \\ \{quang-anh.ta, didier.clouteau,regis.cottereau\}@ecp.fr
}

ABSTRACT. The class of stochastic non-gaussian positive-definite fields with minimal parameterization proposed by Soize (Soize, 2006) to model the elasticity tensor field of a random anisotropic material shows an anisotropy index which grows with the fluctuation level. This property is in contradiction with experimental results in geophysics where the anisotropy index remains limited whatever the fluctuation level. Hence, the main purpose of this paper is to generalize the Soize's model in order to account independently for the anisotropy index and the fluctuation level. It is then shown that this new model leads to major differences in the wave propagation regimes.

RÉSUMÉ. La classe de modèles stochastiques non gaussiens définis-positifs à paramétrage minimal proposé par Soize (2006) pour le champ du tenseur d'élasticité d'un matériau aléatoire anisotrope conduit à des niveaux d'anisotropie du milieu augmentant avec le niveau de fluctuation. Cette propriété étant en contradiction avec les résultats expérimentaux obtenus en géophysique, cet article propose un élargissement du paramétrage du modèle en ajoutant un paramètre contrôlant l'indice d'anisotropie moyen du milieu. On montre alors que ce nouveau modèle conduit à des différences majeures dans le régime de propagation des ondes.

KEYWORDS: wave in random medium, anisotropy, random fields.

MOTS-CLÉS : ondes en milieux aléatoires, anisotropie, champ stochastique.

DOI:10.3166/EJCM.19.241-253 C 2010 Lavoisier, Paris 


\section{Introduction}

When attempting to build predictive models of the dynamic behavior of inhomogenous and complex structures, the account of uncertainties on local properties requires probabilistic approaches. Since the available data is often limited, it is essential to build stochastic fields of elastic properties having a reduced number of parameters so that they can be identified. On the other hand, such a reduced set of parameters may lead to a class of models that is too wide and tend to hide the essential physical phenomena observed in reality. This is particularly salient when the propagation of waves in random medium is of interest since the wave propagation regime can be drastically modified by the type of fluctuation of the elastic properties at stake. The class of non-gaussian positive-definite matrix-valued random field of minimal parameterization proposed by Soize in (Soize, 2006) allows for the modeling of a medium randomly anisotropic. It is fully characterized by a minimal parameterization $\mathcal{P}$ consisting of (i) a mean model (which can be chosen either as isotropic or anisotropic) represented by a mean elasticity tensor $\underline{C}$, (ii) a correlation length vector $\ell$ and (iii) a fluctuation level $\delta$.

One of the key features of this model is that even when considering an isotropic mean medium, the obtained random heterogenous field is locally anisotropic, with a level of anisotropy that can be characterized by the so-called anisotropy index (Arts, 1993; Carcione, 2007). However, statistical analyses on such fields of elastic tensor show that the mean anisotropy index increases almost linearly with the dispersion level. As a consequence, such models are not suited to model geophysical media which can show high fluctuation levels with limited levels of anisotropy.

In this paper, we propose a refined model of non-gaussian random fields of positive-definite elasticity tensors with minimal parametrization and given mean anisotropy index. Section 2 is devoted to the construction of this new random field $\delta_{g}$ and the characterization of its main properties. Using 3D spectral elements, it is shown in section 3 that, given the same level of fluctuation of the elastic properties, the wave propagation pattern is drastically modified when this anisotropy index remains in a range compatible with geophysical data.

\section{Probabilistic model of elastic property fields}

\subsection{Random elastic tensor}

The linear elastic behavior of a material is characterized by the elasticity tensor $\boldsymbol{C}$, linking the stress tensor $\boldsymbol{\sigma}$ and the strain tensor $\boldsymbol{\varepsilon}$. Using Voigt's notation this 4rank tensor can be represented by a 2-rank symmetric positive-definite matrix with 21 independent coefficients for general anisotropic materials. When the material shows local symmetries the number of independent coefficients decreases and reduces to 2 independent coefficients in the isotropic case. Among other choices of that pair 
of coefficients, the tensor $\boldsymbol{C}^{i s o}$ of an isotropic material can be written using the bulk modulus $\kappa$ and shear modulus $\mu$ :

$$
\boldsymbol{C}^{i s o}=3 \kappa S+2 \mu \boldsymbol{D}
$$

where $\boldsymbol{S}$ and $\boldsymbol{D}$ are respectively the so-called spherical tensor and deviatoric tensor defined as: $\boldsymbol{S}=\frac{1}{3}\left(\mathbf{I}_{\mathbf{2}} \otimes \mathbf{I}_{\mathbf{2}}\right)$ and $\boldsymbol{D}=\mathbf{I d}_{\mathbf{6}}-\boldsymbol{S}$ with $\mathbf{I}_{\mathbf{2}}=\left[\begin{array}{lllll}1 & 1 & 1 & 0 & 0\end{array}\right]^{\mathrm{T}}$ and $\mathbf{I} \mathbf{d}_{\mathbf{6}}$ the identity matrix of $\mathbb{M}_{6}(\mathbb{R})$. Since $\{\boldsymbol{S}, \boldsymbol{D}\}$ are orthogonal projectors in the space of real symmetric matrices $\mathbb{M}_{6}^{\mathrm{s}}(\mathbb{R})\left(\boldsymbol{S}^{2}=\boldsymbol{S}, \boldsymbol{D}^{2}=\boldsymbol{D}\right.$ and $\left.\boldsymbol{S} \boldsymbol{D}=0\right)$ and an orthogonal pair for scalar product associated to the Frobenius norm $\left(\|\boldsymbol{S}\|_{\mathrm{F}}=1 ;\|\boldsymbol{D}\|_{\mathrm{F}}=\sqrt{5}\right)$, equation [1] also reads:

$$
C^{i s o}=(\sqrt{3 \kappa} S+\sqrt{2 \mu} D)^{2}
$$

Based on equation [2], we propose to write a random anisotropic elasticity tensor as:

$$
\boldsymbol{C}\left(\boldsymbol{\delta}, \delta_{g}\right)=(\sqrt{3 \kappa(\delta)} \boldsymbol{S}+\sqrt{2 \mu(\delta)} \boldsymbol{D}) \boldsymbol{G}\left(\delta_{g}\right)(\sqrt{3 \kappa(\delta)} \boldsymbol{S}+\sqrt{2 \mu(\delta)} \boldsymbol{D})
$$

in which $\boldsymbol{G}\left(\delta_{g}\right), \kappa(\delta)$ and $\mu(\delta)$ are random variables and $\left(\delta, \delta_{g}\right)$ a pair of dispersion parameters. This model is detailed in sections (2.1.1-2.1.2).

\subsubsection{The anisotropy kernel $\boldsymbol{G}$}

Following (Soize, 2006), the so-called anisotropy kernel $\boldsymbol{G}$ belongs to the set $\mathrm{SG}^{+}$ of all normalized, symmetric, definite-positive real random matrices. This random variable is defined on the probability measure space $(\mathcal{A}, \mathcal{F}, P)$, with values in $\mathbb{M}_{6}^{+}(\mathbb{R})$, parameterized by a unique real positive dispersion parameter $\delta_{g}$. According to (Soize, 2005), the construction by maximizing the entropy leads to the following form of the kernel:

$$
\boldsymbol{G}\left(\boldsymbol{\delta}_{g}\right)=\boldsymbol{L}^{\mathrm{T}}\left(\boldsymbol{\delta}_{g}\right) \boldsymbol{L}\left(\boldsymbol{\delta}_{g}\right)
$$

where $\boldsymbol{L}$ is an upper triangular matrix with entries defined as:

$$
\boldsymbol{L}_{\mathrm{ij}}\left(\delta_{g}\right)= \begin{cases}\frac{\delta_{g}}{\sqrt{7}} \mathcal{G}_{\mathrm{k}}, & \text { for upper extra-diagonal entries } \mathrm{j}>\mathrm{i} \\ \frac{\delta_{g}}{\sqrt{7}} \sqrt{2 h\left(\mathcal{G}_{\mathrm{k}}, \alpha_{\mathrm{i}}\right)}, & \text { for diagonal entries } \mathrm{j}=\mathrm{i}\end{cases}
$$

with:

$-\mathrm{k}$ is a reindexing : $\mathrm{k}=\frac{(14-\mathrm{i})(\mathrm{i}-1)}{2}+\mathrm{j}-\mathrm{i}+1$

$-\mathcal{G}_{\mathrm{k}}$ for $\mathrm{k}=1 . .21$ are 21 independent copies of a normalized centered Gaussian random variable $\mathcal{G}$,

$-h\left(\bullet, \alpha_{i}\right)$ is a non-linear isoprobabilistic transformation that maps a Gaussian scalar variable $(\bullet)$ into a Gamma distributed one. 
$-\alpha_{\mathrm{i}}$ are the parameters of $h\left(\bullet, \alpha_{\mathrm{i}}\right)$ satisfying: $\alpha_{\mathrm{i}}=\frac{7}{2 \delta_{g}^{2}}-\frac{\mathrm{i}-1}{2}$

The dispersion of random matrix $\boldsymbol{G}\left(\boldsymbol{\delta}_{g}\right)$ explicitly depends on $\delta_{g}$ (see (Soize, 2005)):

$$
\frac{E\left\{\left\|\boldsymbol{G}\left(\delta_{g}\right)-\mathbf{I d}_{6}\right\|^{2}\right\}}{6}=\delta_{g}^{2}
$$

\subsubsection{Random isotropic elasticity moduli}

As far as random isotropic elasticity moduli are concerned, the bulk and shear moduli have been chosen since they are the eigenvalues of the elasticity tensor (see for instance (Helbig, 1994; Basser et al., 2007)) and thus lead to a diagonal representation. These moduli are then modeled as independent random variables of strictly positive real value. Applying the maximum entropy principle with given mean values $(\underline{\kappa}, \mu)$ and mean logarithm, leads to two Gamma distributed random variables. In addition, they can be modeled using transforms of 2 independent copies of the Gaussian scalar variable $\mathcal{G}$. It should be noted that, the Gaussian $\mathcal{G}$ is the same as the one constituting the entries of $\boldsymbol{G}$. Hence, by extending the k-subscription used in equation [5] beyond $\mathrm{k}=21$, we can write the 2 elasticity moduli as follows:

$$
\kappa(\delta)=\delta \underline{\kappa} h\left(G_{22}, \delta\right) \quad \text { and } \quad \mu(\delta)=\delta \underline{\mu} h\left(G_{23}, \delta\right)
$$

It is worth noticing that other probability laws such as lognormal could have been chosen, together with correlations between these two gaussian germs.

\subsubsection{Properties of matrix-valued random variable $C$}

Thanks to the knowledge of $\kappa(\delta), \mu(\delta)$ and $\boldsymbol{G}\left(\delta_{g}\right)$, the random elastic tensor $\boldsymbol{C}\left(\delta ; \delta_{g}\right)$ defined in equation [3] has the following properties:

(i) $\boldsymbol{C}\left(\delta ; \delta_{g}\right)$ has an isotropic mean given by:

$$
\underline{C}=3 \underline{\kappa} S+2 \underline{\mu} \boldsymbol{D},
$$

(ii) $\boldsymbol{C}\left(\boldsymbol{\delta}, \delta_{g}\right)$ is a second order random variable:

$$
E\left\{\left\|\boldsymbol{C}\left(\delta, \delta_{g}\right)\right\|_{\mathrm{F}}^{2}\right\} \leq+\infty
$$

(iii) $\boldsymbol{C}^{-1}\left(\delta, \delta_{g}\right)$ is a second order random variable when $\delta^{2}<\frac{1}{2}$ and $\delta_{g}^{2}<\frac{7}{11}$

(iv) The anisotropy level is linearly controlled by $\delta_{g}$.

(v) The global fluctuation of the norm of $C\left(\delta, \delta_{g}\right)$ depends explicitly on on $\delta_{g}$ and $\delta$ as:

$$
\delta_{|C|}^{2}=\frac{E\left\{\|\boldsymbol{C}-\underline{C}\|_{\mathrm{F}}^{2}\right\}}{\|\underline{C}\|_{\mathrm{F}}^{2}}=\delta^{2}+\frac{\delta_{g}^{2}}{7}\left(1+\frac{\operatorname{tr}^{2}(\underline{C})}{\|\underline{C}\|_{\mathrm{F}}^{2}}\right)
$$


The three first properties can easily be worked out and only the last two need to be discussed here. As far as anisotropy level is concerned, several measures can be defined (Arts, 1993; Carcione, 2007). The usual definition uses the distance in the Frobenius norm between the elasticity tensor $\boldsymbol{C}$ and the closest isotropic one denoted by $\boldsymbol{C}_{e q v}^{i s o}$ and defined by:

$$
\boldsymbol{C}_{\text {eqv }}^{i s o}=\left(\boldsymbol{S} \otimes \boldsymbol{S}+\frac{1}{5} \boldsymbol{D} \otimes \boldsymbol{D}\right) \boldsymbol{C}
$$

The anisotropy index, belonging to $[0,1]$ is then defined as:

$$
I_{a}=\sqrt{\frac{\left\|\boldsymbol{C}-\boldsymbol{C}_{e q v}^{i s o}\right\|_{\mathrm{F}}^{2}}{\|\boldsymbol{C}\|_{\mathrm{F}}^{2}}}
$$

Since the mean of this quantity is rather difficult to evaluate analytically, another anisotropy index $\underline{I}_{a}$ is introduced in this paper. Based on decomposition [3], it is defined as the mean square distance between $\boldsymbol{G}\left(\boldsymbol{\delta}_{g}\right)$ and its projection on the isotropic subspace of $\mathbb{M}_{6}^{+}(\mathbb{R})$ :

$$
\overline{\boldsymbol{C}}\left(\boldsymbol{\delta}_{g}\right)=\left(\boldsymbol{S} \otimes \boldsymbol{S}+\frac{1}{5} \boldsymbol{D} \otimes \boldsymbol{D}\right) \boldsymbol{G}
$$

normalized by the square of the norm of the mean value of $\boldsymbol{G}$ :

$$
\underline{I}_{a}=\sqrt{\frac{E\left\{\|\boldsymbol{G}-\overline{\boldsymbol{C}}\|_{\mathrm{F}}^{2}\right\}}{6}}
$$

This new index can be explicitly calculated as a function of $\delta_{g}$ and satisfies the following bounds:

$$
0 \leq \underline{I}_{a}=\sqrt{\frac{19}{21}} \delta_{g} \leq \sqrt{\frac{19}{33}}<0.76
$$

Finally, property (v) is obtained using the expression of the 4th-order tensor of covariance of $\boldsymbol{G}\left(\boldsymbol{\delta}_{g}\right)$ given in (Soize, 2005).

REMARK 1 . When $\underline{I}_{a}=0$ the material is almost surely isotropic. When $\delta=0$, the results on the general random anisotropic tensor given in (Soize, 2005) are retrieved.

EXAMPLE 1. In order to illustrate properties (iv) and (v), a numerical study showing the evolution of the anisotropy index and the global fluctuation $\delta_{C}$ as functions of the pair $\left(\delta, \delta_{g}\right)$ has been performed. The mean elastic tensor $\underline{\boldsymbol{C}}$ corresponds to an isotropic medium with compression wave speed $v_{\mathrm{p}}=1730 \mathrm{~m} / \mathrm{s}$ and shear wave speed $v_{\mathrm{s}}=1000 \mathrm{~m} / \mathrm{s}$. The statistic evaluations performed using 1000 samples for each configuration of $\left(\delta, \delta_{g}\right)$ are shown to be in good accordance with the theoretical estimates. For instance, only the comparision of $\delta_{|C|}$ is displayed in (Figure 1-a). On the other hand, (Figure 1-b) shows that the new index $\underline{I}_{a}$ is almost equivalent to the mean of the classical one (although the fact that the one given by equation [12] seems to be slightly dependent on $\delta$ ). 


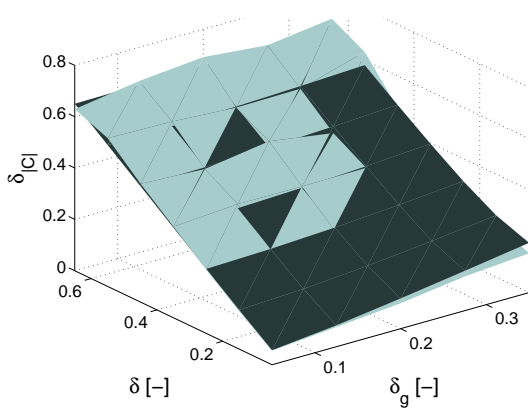

(a)

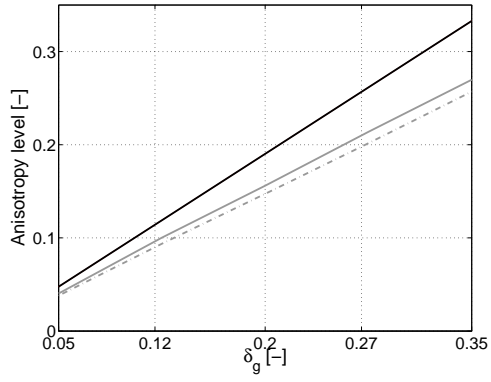

(b)

Figure 1. (a) Fluctuations $\delta_{|C|}$ of elasticity tensor: theorical estimates (dark) and statistic ones (light). (b) New index $\underline{I}_{a}$ (black solid line) and mean values of the clasical one (gray dash line for $\delta=0.65$, gray solid line for $\delta=0.05$ )

\subsection{Stochastic field of elasticity tensor}

Up to now, only the variability of the elasticity tensor at a given point has been accounted for. In order to introduce the spatial variability of this mechanical property, the present section discusses the construction of a model of the stochastic field of elasticity tensor based on the probabilistic model developed in the previous section. Let $\boldsymbol{\Omega}=\left\{\boldsymbol{x} \mid \boldsymbol{x} \in \mathbb{R}^{3}\right\}$ be the physical domain, equipped with a Cartesian reference frame $\left\{\boldsymbol{i}_{1}, \boldsymbol{i}_{2}, \boldsymbol{i}_{3}\right\}$, and occupied by an inhomogeneous elastic material. The associated stochastic field model of elasticity tensor $\left\{\mathbb{C}\left(\delta ; \delta_{g} ; \ell\right) \mid \boldsymbol{x} \in \boldsymbol{\Omega}\right\}$, defined on the probability measure space $(\mathcal{A}, \mathcal{F}, P)$, indexed on $\boldsymbol{\Omega}$, with values in $\mathbb{M}_{6}^{+}(\mathbb{R})$, can then be formulated as follows:

$$
\begin{gathered}
\left\{\mathbb{C}\left(\boldsymbol{x} ; \delta, \delta_{g} ; \boldsymbol{\ell}\right)=(\sqrt{3 \kappa(\boldsymbol{x} ; \delta ; \boldsymbol{\ell})} \boldsymbol{S}+\sqrt{2 \mu(\boldsymbol{x} ; \delta ; \boldsymbol{\ell})} \boldsymbol{D}) \boldsymbol{G}\left(\boldsymbol{x} ; \delta_{g} ; \boldsymbol{\ell}\right)\right. \\
(\sqrt{3 \kappa(\boldsymbol{x} ; \delta ; \ell)} \boldsymbol{S}+\sqrt{2 \mu(\boldsymbol{x} ; \delta ; \ell) D})\} \text { a.s. }
\end{gathered}
$$

where $\ell=\left(\ell_{1}, \ell_{2} \ell_{3}\right)$ is a vector of correlation lengths in the three spatial directions. The evolution from equation [3] to equation [16] is done by replacing, in the formulation of the kernel $\boldsymbol{G}$ and of the isotropic elastic modulus $\kappa, \mu$, the 23 independent copies $\left\{G_{\mathrm{k}} \mid \mathrm{k} \in\{1,2, \ldots, 23\}\right\}$ of a Gaussian normalized random variable by 23 independent copies $\left\{\boldsymbol{G}_{\mathrm{k}}(\boldsymbol{x} ; \boldsymbol{\ell}) \mid \boldsymbol{x} \in \boldsymbol{\Omega} ; \mathrm{k} \in\{1,2, \ldots, 23\}\right\}$ of a stochastic Gaussian field $\{\boldsymbol{G}(\boldsymbol{x} ; \boldsymbol{\ell}) \mid \boldsymbol{x} \in \boldsymbol{\Omega}\}$ indexed on $\boldsymbol{\Omega}$ with values in $\mathbb{R}$. This germ Gaussian field is of secondorder, homogeneous with a correlation structure defined by the following correlation function (see, (Soize, 2006; Arnst, 2007; Popescu, 1995), for more details):

$$
R_{\mathcal{G}}(\boldsymbol{\eta} ; \boldsymbol{\ell})=E\{\boldsymbol{G}(\boldsymbol{x} ; \boldsymbol{\ell}) \boldsymbol{G}(\boldsymbol{x}+\boldsymbol{\eta} ; \boldsymbol{\ell})\}=\rho\left(\eta_{1}, \ell_{1}\right) \rho\left(\eta_{2}, \ell_{2}\right) \rho\left(\eta_{3}, \ell_{3}\right)
$$


where $\rho(\eta ; \ell)$ is chosen as a squared cardinal sine:

$$
\rho(\eta ; \ell)=\frac{4 \ell^{2}}{\pi^{2} \eta^{2}} \sin ^{2}\left(\frac{\pi \eta}{2 \ell}\right)
$$

This stochastic field $\left\{\mathbb{C}\left(\boldsymbol{x} ; \boldsymbol{\delta}, \boldsymbol{\delta}_{g} ; \ell\right)\right\}$ is mean-square continuous with almost surely continuous samples. Following (Soize, 2006), it can be shown that taking the restriction of this field on a bounded domain leads to a second order solution of the related stochastic boundary value problem.

EXAMPLE 2. As an example, a soil cube filling the physical domain $\left\{\boldsymbol{x} \in \boldsymbol{\Omega}_{0} \subset\right.$ $\left.\boldsymbol{\Omega} \mid-200 \mathrm{~m} \leq x_{1}, x_{2} \leq 200 \mathrm{~m} ;-400 \mathrm{~m} \leq x_{3} \leq 0 \mathrm{~m}\right\}$ is considered now. The material has a constant bulk density $\rho_{v}=2000 \mathrm{~kg} / \mathrm{m}^{3}$. The mean model consists in a homogeneous isotropic elastic material defined in (Example 1). A simulation by the spectral representation approach (see, (Shinozuka et al., 1996), for instance.) is then performed. The mapping of term $C_{11}$ of a sample of the field $\mathbb{C}\left(x ; \delta, \delta_{g} ; \boldsymbol{\ell}\right)$ with $\delta=0.6 ; \delta_{g}=0.15 ; \ell_{1}=\ell_{2}=50 \mathrm{~m}$ and $\ell_{3}=20 \mathrm{~m}$ is shown in (Figure 2 -a). The shorter correlation length along the vertical axis is clearly visible on this chart. In Figure2-b, a fair matching between the theorical unidimensional correlation function given by equation [18] and the ones obtained by spatial mean is observed. Another remark is that despite the isotropic mean behavior, the elastic tensor is anisotropic almost everywhere. For instance, the elasticity tensor at the point $\left\{x_{1}=x_{2}=x_{3}=0\right\}$ of the given field sample is:

$$
C(\mathbf{0}, \ldots)=\left(\begin{array}{cccccc}
6.369 & 0.461 & 0.880 & 0.671 & -0.303 & -0.178 \\
- & 3.949 & 1.403 & 0.039 & -0.405 & -0.513 \\
- & - & 4.394 & -0.142 & -0.152 & -0.662 \\
\mathrm{~S} & - & - & 3.091 & -1.072 & -0.081 \\
- & \mathrm{Y} & - & - & 2.036 & 0.224 \\
- & - & \mathrm{M} . & - & - & 2.047
\end{array}\right)\left[\times 10^{9} \mathrm{~Pa}\right]
$$

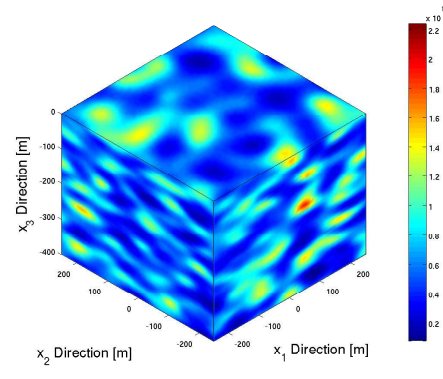

(a)

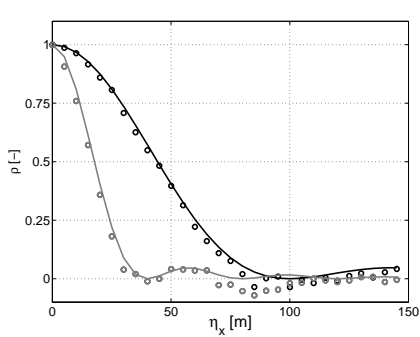

(b)

Figure 2. A sample of stochastic elasticity tensor field: (a) Mapping of $C_{11}$. (b) Theorical (solid lines) and observed (circles) correlation structures in $\boldsymbol{i}_{1}-$ (black) and $\boldsymbol{i}_{3}-$ (gray) directions 


\section{Effect of the random field model on wave propagation pattern}

In this section, the influence of the stochastic model of a random anisotropic media on the wave propagation pattern is characterized by means of numerical simulations. Since the goal is to characterize the wave propagation pattern in a random media beyond the mean free path, a numerical method is required, that is able to account for several wavelengths and correlation lengths in all spatial directions. Moreover, since the multiple scattering pattern drastically changes between two and three dimensional cases, 3D simulations are targeted. In order to meet both a high efficiency and a controlled numerical error, the Spectral Finite Element Method has been chosen. In particular, the SPEC software developped by the Seismology Group of Institut de Physique du Globe de Paris (Festa et al., 2005) has been modified in order to account for anisotropic heterogeneous fields of elastic tensors. It is worth noticing that in this section only samples of random media will be considered and no real statistical analyses will be performed. However, since the elastic waves are traveling through a statistically homogeneous random media, the wave pattern obtained after several wavelengths and correlation lengths is expected to show common statistical properties weakly dependent on the sample of the random media. In particular, this property has been observed when considering the multiple scattering of seismic wave fields on a random distribution of buildings (Clouteau et al., 2001).

The strong formulation of the elastic wave propagation in an anisotropic media $\Omega$ consists in solving for $\boldsymbol{u}$ the Navier equation $\forall t \in[0 ; T]$ :

$$
\operatorname{Div}(\boldsymbol{C}(\boldsymbol{x}) \boldsymbol{\varepsilon}(\boldsymbol{u}(\boldsymbol{x} ; t)))+\boldsymbol{f}(\boldsymbol{x}, t)=\rho_{v} \frac{\partial^{2}}{\partial t^{2}} \boldsymbol{u}(\boldsymbol{x} ; t)
$$

together with proper boundary and initial conditions. $\boldsymbol{C}$ is a sample of the random field of anisotropic elastic tensor and $\rho_{v}$ is the bulk density. As far as the Spectral Finite Element Method is concerned, the related weak formulation is considered $\forall t \in[0 ; T]$ and $\forall \boldsymbol{w} \in V(\Omega)$ :

$$
\int_{\Omega}^{*}\left(\rho_{v} \frac{\partial^{2} \boldsymbol{u}}{\partial t^{2}}(t) \cdot \boldsymbol{w}+\boldsymbol{C}^{*}(\boldsymbol{x}) \boldsymbol{\varepsilon}(\boldsymbol{u}(t)): \boldsymbol{\varepsilon}(\boldsymbol{w})-\boldsymbol{f}(\boldsymbol{x}, t) \cdot \boldsymbol{w}\right) d \Omega=0
$$

where $\boldsymbol{C}^{*}=\boldsymbol{C}$ in the domain of interest $\Omega$ and where $\boldsymbol{C}^{*}$ corresponds to a modified divergence operator $\widetilde{\text { Div }}$ inside the so-called PML (i.e. Perfectly Matched Layer) $\Omega^{*} \backslash \Omega$. Indeed, the PML can be viewed as an anisotropic dispersive material. In addition, it is worth noticing that such PML are applicable to inhomogeneous media but can become instable for anisotropic materials (Bécache et al., 2003). As a consequence, they have to be modified using a multiaxial damping (Meza-Fajardo et al., 2008) leading to stable but not perfectly matched layers. This is a serious drawback when homogeneous material are sought for. It is less important for a strongly inhomogeneous media since perfectly or non perfectly matched layers are both wrong in this case. The development of equivalent boundary conditions in such media is still a pending question to the authors' knowledge. 


\subsection{Elastic waves in homogeneous anisotropic media}

In order to validate the Spectral Finite Element code for anisotropic media, the wave propagation in a homogeneous anisotropic medium is first studied. Indeed, for homogeneous physical properties in $\mathbb{R}^{3}$, equation [20] can be analytically solved for plane waves solutions:

$$
\boldsymbol{u}(\boldsymbol{x}, t)=\hat{\boldsymbol{u}}_{ \pm}(\boldsymbol{s}, \omega) \exp (i \omega(\boldsymbol{s} \cdot \boldsymbol{x} \pm t))
$$

where $s$ is the slowness vector and $\omega$ the circular frequency. It leads to the Christoffel eigenvalues problem (Auld, 1973) for $\boldsymbol{s}$. These solution vectors belong to three slowness surfaces which represent the inverses of phase velocity as functions of the wave propagation direction. These 3 surfaces stand for 1 quasi-longitudinal mode ( $q P$ mode) and 2 quasi-transverse modes ( $q S$-modes). Apart from the slowness (or phase velocity), each of these propagation modes can be characterized by a group velocity with a closed-form expression, which lead to a set of three group velocity surfaces. This analytical solution can be used to validate the wave propagation in an unbounded homogenous elastic tridimensional domain under a point source excitation. Indeed, the instantaneous wavefronts are obtained by multiplying group velocity surface by travel time.

EXAMPLE 3. We consider in this example an unbounded homogenous domain with an elasticity tensor given by formula [19]. Slowness surfaces are then shown in (Figure 3). In this picture, some key characters of the anisotropy can be revealed. The two $q S$-modes differ leading to a shear wave splitting which does not occur in the isotropic case. Moreover, the 3 surface sheets have irregular shapes corresponding to an a priori triclinic elasticity tensor. On the other hand, simple wavefront predictions can be represented by wavefront curves in (Figure 4). These curves are peformed by intersecting group velocity surfaces with the 3 planes $x_{1}=0, x_{2}=0, x_{3}=0$ and then scaling by the travel time $(t=0.59 \mathrm{~s}$ in this case). We can remark that $q S$-mode group velocity surfaces are multi-valued along directions corresponding to locally concave slowness surfaces (Auld, 1973; Cerveny, 2001). Also in (Figure 4), theorical wavefronts are then superposed with the velocity module snapshots of a wave propagation simulated by spectral finite elements approach. In the simulation, the physical domain defined by $-1000 \mathrm{~m} \leq x_{1}, x_{2} \leq 1000 \mathrm{~m}$ and $-2000 \mathrm{~m} \leq x_{3} \leq 0 \mathrm{~m}$, is surrounded by PML domains.

\subsection{Waves in random anisotropic media}

We now consider two samples of a random media having the same: mean homogeneous isotropic elastic properties, fluctuation level $\delta_{|C|}$ and correlation length vector $\ell$, but different values for the dispersion parameters $\delta$ and $\delta_{g}$ in order to observe the influence of these parameters on the wave propagation pattern.

EXAMPLE 4. The domain of interest is such that $-1500 \mathrm{~m} \leq x_{1}, x_{2} \leq 1500 \mathrm{~m}$ and $-400 \mathrm{~m} \leq x_{3} \leq 0 \mathrm{~m}$. The mean wave velocities are $v_{\mathrm{p}}=1730 \mathrm{~m} / \mathrm{s}$ and $v_{\mathrm{s}}=1000 \mathrm{~m} / \mathrm{s}$. 

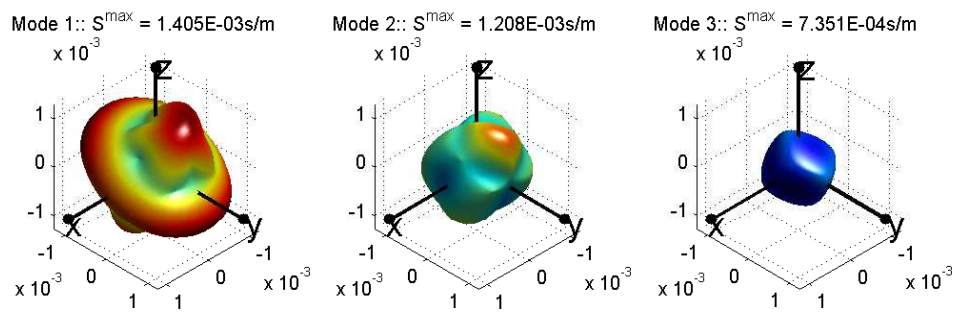

Figure 3. Slowness surface. From left to right: $2 q S$-modes and $1 q P$-mode

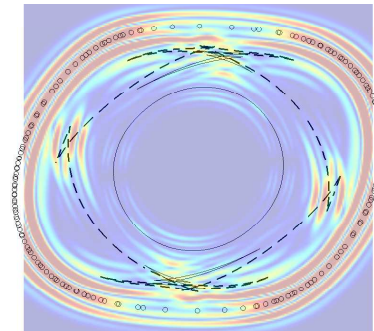

(a)

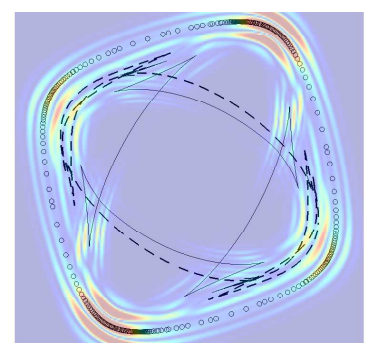

(b)

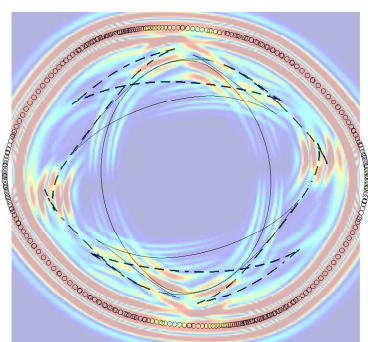

(c)

Figure 4. Snapshots of the simulated velocity module $v s$. theorical wavefronts at $t=$ 0.59s: (a) Plane $x_{3}=0$. (b) Plane $x_{2}=0$. (c) Plane $x_{1}=0$ in which the $q S 1-, q S 2-$, $q P$ - theorical modes are displayed respectively in dash line, solid line and o-marked curves

Free surface boundary conditions are applied on top $x_{3}=0$ and PML conditions on the other sides of the box. A point source is located at point $x_{o}=(-1400,0,0)$ on the free surface. The time history of the force is a Ricker with a characteristic frequency equal to $10 \mathrm{~Hz}$. The three correlation lengths are equal to $100 \mathrm{~m}$. As a consequence, the dominant wavelength is of the order of the correlation length and the domain characteristic size is about ten times the wavelength. As far as the amplitude of the fluctuation is concerned, the global fluctuation level on the elastic tensor is set to $\delta_{|C|}=$ 0.49 . The dispersion parameters are set to $\delta=0 ; \delta_{g}=0.6$ for the first model, which corresponds to the original model proposed in (Soize, 2006). These parameters are set to $\delta=0.47 ; \delta_{g}=0.17$ for the second model which corresponds to the upper limit of the anisotropic index observed in geophysics (Vernik et al., 1997). The amplitudes of the particle velocity on the free surface are shown in (Figure 5) for three different time steps and for the two models. Although $\delta_{|C|}$ is the same in the two cases, the wave propagation patterns are very different. In the case of a high anisotropic level $\left(\delta_{g}=0.6\right)$ the diffusion pattern corresponds to a strong diffusion of the wave field whereas in the weakly anisotropic case $\left(\delta_{g}=0.17\right)$ the wave field seems much more 
localized in space and in time. Scattering at given locations can be observed. These results indicate that these two models lead to different propagation regimes which have to be charaterized in more details.
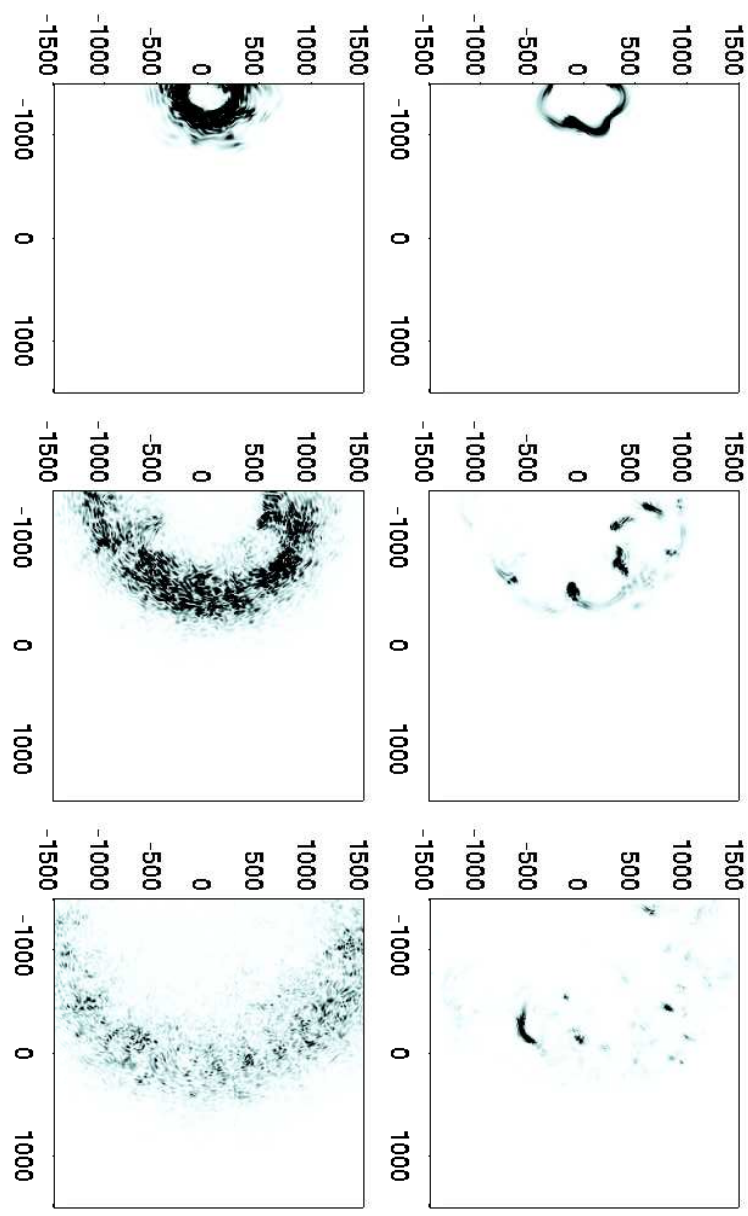

Figure 5. Time evolution (downward) of wavefronts on the free surface for $\delta_{|C|}=0.49$. (left) $\delta=0 ; \delta_{g}=0.6$, (right) $\delta=0.47 ; \delta_{g}=0.17$

\section{Conclusions}

In this paper a refinement of the non-gaussian random field of anisotropic elastic tensor with minimum parameters (Soize, 2006) has been proposed in order to control the anisotropy index independently from the fluctuation level. This new model is based on the splitting of the elastic tensor into isotropic and anisotropic parts which are modeled independently. This new model introduces a single new parameter which 
has been shown to depend linearly on a modified mean anisotropy index. Monte Carlo simulations have shown that this modified index is almost equivalent to the classical one.

Numerical simulations have been performed using time domain 3D Spectral Finite Element on large samples of the proposed random fields. It has been demonstrated that given a large fluctuation level, the pattern of the wave field after a few wavelengths is highly dependent on the mean anisotropy index which can now been controlled independently from the fluctuation level.

It is worth noticing that correlations between the isotropic elastic moduli and the bulk density can easily be accounted for in the proposed model together with different correlation lengths for the random anisotropy and the isotropic elastic moduli.

\section{References}

Arnst M., Inversion of probabilistic models of structures using measured transfer function, $\mathrm{PhD}$ thesis, Ecole Centrale Paris, France, 2007.

Arts R. J., Étude de l'élasticité anisotrope générale dans les roches par propagation des ondes., $\mathrm{PhD}$ thesis, Université de Pierre et Marie Curie, 1993.

Auld B. A., Acoustic fields and waves in solids, vol. 1-2, John Wiley \& Sons, 1973.

Basser P., Pajevic S., "Spectral decomposition of a 4th-order covariance tensor : Application to diffusion tensor MRI”, Signal Processing, vol. -, n 87, p. 220-236, 2007.

Bécache E., Fauqueux S., Joly P., " Stability of perfectly matched layers, group velocities and anisotropic waves", Journal of Computational Physics, vol. -, n 188, p. 399-433, 2003.

Carcione J.-M., Wave Fields in Real Media : Wave Propagation in Anisotropic, Anelastic, Porous and Electromagnetic Media, vol. 38 of Hanbook of Geophysical Exploration : Seismic Exploration, second : revised and extended edn, Elsevier, 2007.

Cerveny V., Seismic ray theory, Cambridge University Press, 2001.

Clouteau D., Aubry D., “ Modification of the ground motion in dense urban areas", Journal of Computational Acoustics, vol. 9, n 4, p. 1659-1675, 2001.

Festa G., Vilotte J., “ The Newmark scheme as a velocity-stress time staggering: An efficient PML for spectral element simulations of elastodynamics", Geophysical Journal International, vol. 161, n 3, p. 789-812, 2005.

Helbig K., Foundations of Anisotropy for Exploration Seismics, Elsevier, 1994.

Meza-Fajardo K., Papageorgiou A., " A Nonconvolutional, Split-Field, Perfectly Matched Layer for Wave Propagation in Isotropic and Anisotropic Elastic Media: Stability Analysis", Bulletin of the Seismological Society of America, vol. 98, n 4, p. 1811-1836, 2008.

Popescu R., Stochastique variability of soil propeties : data analysis, digital simulation, effect on system behavior, $\mathrm{PhD}$ thesis, Princeton University, USA, 1995.

Shinozuka M., Deodatis G., "Simulation of multi-dimensional Gaussian stochastic fields by spectral representation”, Applied Mechanics Review, vol. 1, n 49, p. 29-53, 1996. 
Soize C., " Random matrix theory for modeling uncertainties in computational mechanics", Computer Methods in Applied Mechanics and Engineering, vol. -, $\mathrm{n}^{\circ}$ 194, p. 1333-1366, 2005.

Soize C., "Non-Gaussian positive-definite matrix-valued random fields for elliptic stochastic partial differential operators", Computer Methods in Applied Mechanics and Engineering, vol. 1-3, n 195, p. 26-64, 2006.

Vernik L., Liu X., "Velocity anisotropy in shales: A petrophysical study”, Geophysics, vol. 62, p. 521-532, 1997. 
